\title{
Organ Segmentation with Level Sets Using Local Shape and Appearance Priors
}

\author{
Timo Kohlberger ${ }^{1}$, M. Gökhan Uzunbaş ${ }^{1}$, Christopher Alvino ${ }^{1}$, Timor Kadir ${ }^{2}$, \\ Daniel O. Slosman ${ }^{3}$, and Gareth Funka-Lea ${ }^{1}$ \\ 1 Siemens Corporate Research, Imaging and Visualization Dept., Princeton, USA \\ 2 Siemens Healthcare Molecular Imaging, Oxford, UK \\ 3 Clinic Generale-Beaulieu, Geneva, Switzerland
}

\begin{abstract}
Organ segmentation is a challenging problem on which recent progress has been made by incorporation of local image statistics that model the heterogeneity of structures outside of an organ of interest. However, most of these methods rely on landmark based segmentation, which has certain drawbacks. We propose to perform organ segmentation with a novel level set algorithm that incorporates local statistics via a highly efficient point tracking mechanism. Specifically, we compile statistics on these tracked points to allow for a local intensity profile outside of the contour and to allow for a local surface area penalty, which allows us to capture fine detail where it is expected. The local intensity and curvature models are learned through landmarks automatically embedded on the surface of the training shapes. We use Parzen windows to model the internal organ intensities as one distribution since this is sufficient for most organs. In addition, since the method is based on level sets, we are able to naturally take advantage of recent work on global shape regularization. We show state-of-the-art results on the challenging problems of liver and kidney segmentation.
\end{abstract}

\section{Introduction}

Level set methods have many strengths that make them suitable for general organ segmentation, which include the ability to naturally represent complex shapes non-parametrically, and the ability to incorporate powerful shape models 410155. Until recently, most level set-based segmentation methods have focused on global data likelihood models [16] and global priors on contours such as surface area penalty [9. Unfortunately, such global models do not fully exploit the fact that surrounding image intensities and local organ curvatures vary in a predictably local fashion, e.g., in the segmentation of livers in computed tomography (CT) images in which the outside intensities and contour curvatures are naturally heterogeneous.

Thus many mechanisms to allow for local evolution have been introduced both in the computer vision literature [13, and in the medical imaging literature 11612. Some methods allow for local intensity models in a level set framework, but not in a way that allows position dependent knowledge to be 
accounted for [13]. Other methods use landmarks to represent the organ boundary $86[12] 11$, and allow incorporation of shape through active shape models [3]. While these methods have achieved significant progress on difficult organ segmentation problems such as the liver, no completely satisfactory solution exists due to high variability in anatomical shape, from disease, and in acquisition protocol. Additionally, segmenting only within the space of shapes allowable by ASM's is a limiting factor since ASM's tend to naturally produce shapes that smooth out naturally high curvature structures. Furthermore, determining segmentation boundaries in between points requires, as a final step, choosing some method of interpolating between landmarks [11].

In this paper, we introduce a novel efficient point tracking mechanism into standard level set evolution, which allows us to formulate the problem as a maximum a posteriori estimation problem with both a local likelihood model for the data term as well as a local prior model for the surface area penalty term. In doing this, we extend the work of [14, where point tracking is used to define correspondence during level set evolution, by simplifying the tracking for greater efficiency at the cost of maintaining an approximate correspondence that is still sufficient for segmentation. In addition, we show how introducing shape-guidance into this level set framework is natural and we can take advantage of recent work on shape-guided level set methods (see [5] and references therein.) We validate the efficacy of this point tracking method on complex organ segmentation problems such as liver and kidney segmentation, while also showing that it is very efficient. We show that the method produces segmentations with state of the art overlap and surface error statistics, as made popular in the MICCAI 2007 Liver challenge.

\section{Key Point Tracking on Evolving Zero Level Sets}

To facilitate the application of local prior statistics and constraints it is necessary to track explicit points on the evolving surface. This is done as follows.

Consider a set of discrete points $\left\{x_{i}(t)\right\}_{i=1, \ldots, N}$ defined on the initial zero level set of a signed distance function $\phi(\mathbf{x}, t), \mathbf{x} \in \Omega \subset \mathbb{R}^{3}$ at $t=0$. As explained in 14] and the references therein, those can be tracked along with the evolution of the zero level set by mapping them along its outer normal $\mathbf{n}\left(\mathbf{x}_{i}(t), t\right)=$ $-\nabla \phi\left(\mathbf{x}_{i}(t), t\right) /\left|\nabla \phi\left(\mathbf{x}_{i}(t), t\right)\right|$ by the amount of the level set speed $\Delta \phi(\cdot, t+1)$ :

$$
\mathbf{x}_{i}(t+1) \leftarrow \mathbf{x}_{i}(t)+\Delta \phi\left(\mathbf{x}_{i}(t), t+1\right) \mathbf{n}\left(\mathbf{x}_{i}(t), t\right) .
$$

This is to be carried out for each level set update, see Fig. 1 1 for an example.

However, in the case of a narrow-band implementation, especially when the speed of the level set is high, the update of the key point $\mathbf{x}_{i}(t)$ might locate the new position $\mathbf{x}_{i}(t+1)$ such that it lies outside of the band of $\phi(\cdot, t+1)$. In order to handle such cases, we linearly search along the normal of $\phi(\cdot, t)$ to a pre-defined extent, i.e. along the line $\mathbf{x}_{i}(t)+\tau \mathbf{n}_{\mathbf{0}}\left(\mathbf{x}_{i}(t), t\right)$, with $\tau>0$. (We found sampling $\tau$ at intervals of one voxel length to be a reasonable value.) By this we bring down the fraction of "lost" key points typically below $0.1 \%$. 
Furthermore, due to numerical inaccuracies in the update scheme (1), the key points may deviate from the zero levelset after some iterations. For such points, we apply an additional correction scheme:

$$
\mathbf{x}_{i}(t) \leftarrow \mathbf{x}_{i}(t)+\phi\left(\mathbf{x}_{i}(t), t\right) \mathbf{n}_{\mathbf{0}}\left(\mathbf{x}_{i}(t), t\right),
$$

which guarantees that the updated key points are always on the zero level.

In the following, we use the described algorithm to establish shape correspondences between the initial shape $\phi(\cdot, 0)$, which we will refer to as reference shape, and the segmenting shape $\phi(\cdot, t)$. Obviously, because of neglecting the tangential evolution of similarity features, such a correspondence is only approximate, but will be sufficient in the following if the reference and the target shape are not too dissimilar and are roughly rigidly aligned. Compared to Poisson-equation-based methods, cf. 14, this method has the advantage of being computationally much more efficient. For example, tracking 3126 key points in the case shown in Fig. 1] along with a narrow-band consisting of 35000 voxels added only $2.3 \%$ to the average computation time of a level set update.
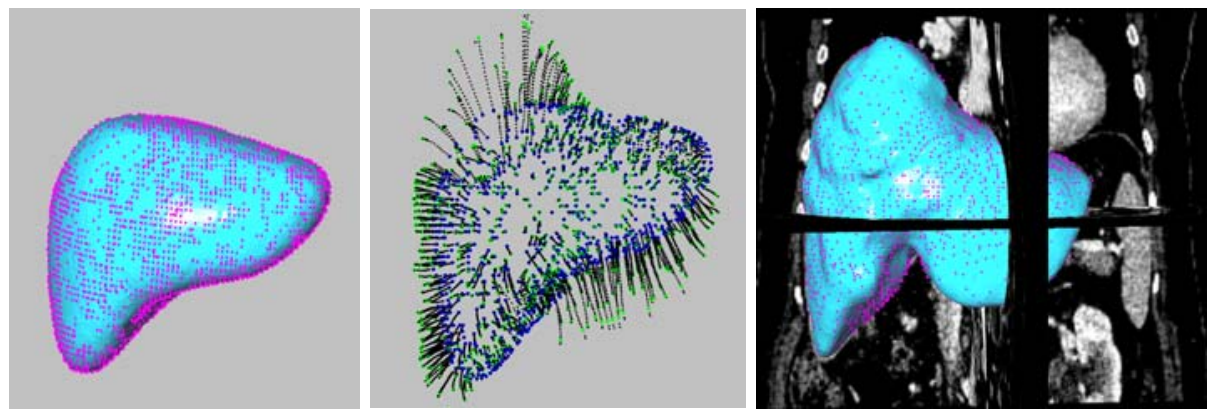

Fig. 1. (a) Example of initial key points on an initial zero level being rigidly aligned to the organ to segment. (b) Evolution of each key point along the normals of the zero level sets while carrying out a standard data-driven segmentation iteration. (c) Result after 200 level set/key point tracking iterations. Given a sufficient similarity of the initial shape as well as an initial alignment, tracking points along the normal at each iteration is sufficient to retrieve an approximate shape correspondence.

\section{Levelset Segmentation Driven by Local Feature Models}

Given a set of explicit points that have correspondences to the initial shape, we can assign locally measured features of different level set evolutions to one common reference shape. Such measurements from the segmentation of a set of training samples are accumulated on the reference shape to deduce localized feature models and it is these models which then are introduced as priors in the segmentation approach. 


\subsection{Local Intensity Statistics}

To build a localized intensity prior, we carried out a standard data-driven segmentation approach 2 2 on expert-generated binary annotation masks of the intended organs, while employing the scheme presented in section 2 to track the key points in parallel. Thereby the initial/reference shape was a typical shape of the organ and rigidly aligned to each of those masks.

Once the zero level set had converged at or near the binary edge, at each key point, we sampled the local intensity outside the contour from the intensity volume being aligned with the annotation mask. Specifically, we convolved with small Gaussian windows which were centered at each point separately for inside and outside voxels, which in the latter case reads:

$$
I_{j, \rho}^{\text {out }}\left(\mathbf{x}_{i}\right)=\frac{\int_{\Omega_{i}} g_{\rho}\left(\mathbf{x}_{i}-\mathbf{y}\right)\left(1-H\left(\phi_{i}(\mathbf{y})\right)\right) I_{j}(\mathbf{y}) d \mathbf{y}}{\int_{\Omega_{i}} g_{\rho}\left(\mathbf{x}_{i}-\mathbf{y}\right)\left(1-H\left(\phi_{i}(\mathbf{y})\right)\right) d \mathbf{y}}
$$

with $g_{\rho}(\mathbf{x})=\exp \left(-\mathbf{x}^{\top} \mathbf{x} /\left(2 \rho^{2}\right)\right), I_{j}(\mathbf{x})$ referring to the value of the intensity volume (not the segmentation mask) at $\mathbf{x}$, and $H$ denoting the Heaviside function.

In a next step, for each key point on the reference shape, we accumulated these samples across the training cases by fitting Gaussian distributions, whose spatially dependent parameters were determined according to:

$\mu_{\text {out }}\left(\mathbf{x}_{i}\right)=\frac{1}{L} \sum_{j=1}^{L} I_{j, \rho}^{\text {out }}\left(\mathbf{x}_{i}\right), \quad \sigma_{\text {out }}^{2}\left(\mathbf{x}_{i}\right)=\frac{1}{L-1} \sum_{j=1}^{L}\left(\mu_{\text {out }}\left(\mathbf{x}_{i}\right)-I_{j, \rho}^{\text {out }}\left(\mathbf{x}_{i}\right)\right)^{2}$.

See Fig. 2(a)-(b) for an example on the liver.

Subsequently, during the course of the level set evolution, the such inferred Gaussian likelihoods are not only available at tracked key point locations, but can be interpolated to any voxel in the narrow-band. In case of a simple nearestneighbor interpolation, the prior probability of observing an intensity $I(\mathbf{x})$ at a location $\mathbf{x}$ near or at the zero level can thus be approximated by

$$
p_{\text {out }}(I(\mathbf{x}) \mid \mathbf{x}):=\frac{1}{\sqrt{2 \pi \sigma_{\text {out }}^{2}\left(\mathbf{x}_{l(x)}\right)}} \exp \left(-\frac{\left(I(\mathbf{x})-\mu_{\text {out }}\left(\mathbf{x}_{l(x)}\right)\right)^{2}}{2 \sigma_{\text {out }}^{2}\left(\mathbf{x}_{l(x)}\right)}\right),
$$

with $l(x)=\arg \min _{i=1, \ldots, N}\left\|\mathbf{x}-\mathbf{x}_{i}\right\|_{L_{2}}^{2}$ being the closest key point with regards to the Euclidean distance. With respect to implementing the latter, we found [1] to provide a very efficient solution.

\subsection{Local Mean Curvature Constraint}

Local correspondences between the evolving and a reference shape not only enable spatially dependent intensity priors, but also allow for a spatially dependent weighting of feature measurements, such as the mean curvature.

Given the tracked key points at the boundaries of ground truth annotation masks, as described in the previous section, one can similarly sample local mean curvatures and average them over the set of training cases, which yields an average mean curvature for each key point (see Fig. 2(c) for an example): 

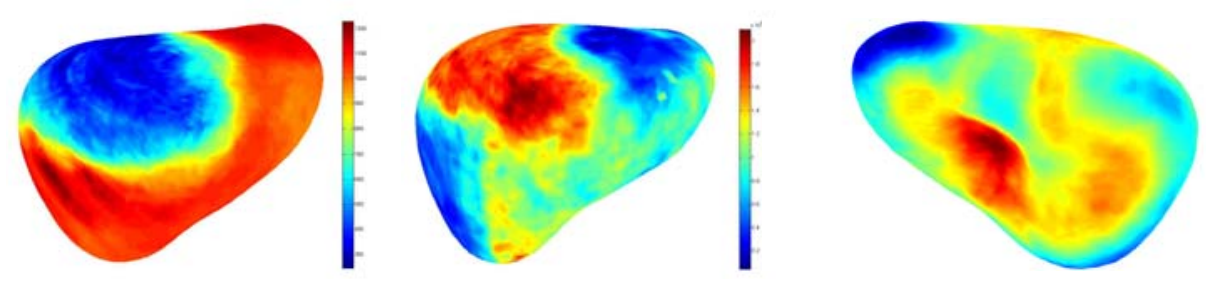

Fig. 2. Means (a) and variances (b) of the outward-bound local intensities across 100 liver shapes, which were traced back to the shown reference shape. (c) Average measured mean curvatures. Despite high shape variability, sharp correspondences are retrieved by the proposed key point tracking method, which is visible especially at the rib region in $(\mathrm{a})+(\mathrm{b})$, and at the liver tips in (c).

$$
\kappa_{P}\left(\mathbf{x}_{i}\right)=\frac{1}{L} \sum_{j=1}^{L} \operatorname{div} \frac{\nabla \phi_{j}\left(\mathbf{x}_{i}\right)}{\left|\nabla \phi_{j}\left(\mathbf{x}_{i}\right)\right|} .
$$

Instead of inferring prior densities, which would involve second-order derivates of $\phi$ in the energy and thus numerically problematic fourth-order derivatives in the Euler-Lagrange equations, here we propose to introduce the spatiallydependent prior by modulating the weight of the area term, cf. 2], in the energy:

$$
E_{C}(\phi)=\int_{\Omega} \omega_{C}(\mathbf{x})|\nabla H(\phi)| d \mathbf{x}, \quad \text { with } \quad \omega_{C}(\mathbf{x})=\frac{\xi}{1+\nu \kappa_{P}^{2}(\mathbf{x})},
$$

with a parameter $\nu$ controlling the amount of weight variation and $\xi$ denoting a global weigth.

Thus, we penalize surface area of the segmenting contour more strongly at locations when low curvatures were observed on the training contours, and viceversa for high curvatures. For example, by this the curvature term can be weak at the tip regions of a liver shape, while enforcing low curvatures everywhere at relatively straight regions. See Fig. 3 for examples on the CT liver.

\section{Organ Segmentation Approach}

In the following we combine the localized feature priors with established ideas in order to obtain a highly accurate, robust and generic organ segmentation approach. Thereby our objects of study are the segmentation of the liver and the right kidney in $3 \mathrm{D} \mathrm{CT}$ images.

\subsection{Local Models for the Liver and the Kidney}

We applied the technique described in Sec. 3.1 on 100 liver and 20 right kidney ground truth segmentations, in order to estimate the local mean and variance of the intensities inside and outside the segmentation boundary. For the outside intensities, we obtained maps which clearly reflect heterogeneous intensity regions of the neighboring tissue classes, see Fig. 2(a)-(b). 
By contrast, the accumulated inside measurements turned out to be very homogeneous per training case, but to vary in a range of about 150 Hounsfield units across all training cases. Both observations are in line with the fact that the tissues of both organs exhibit relatively homogeneous CT intensities, while their mean fluctuates with the concentration of contrast agent. In order to account for these effects, we chose an adaptive region-based Parzen density model, cf. e.g. 4, for the observed intensities inside the organ:

$$
p_{i n}(I(\mathbf{x}) \mid \phi):=\sum_{l=I_{\min }}^{I_{\max }} \frac{h(l)}{H} g_{\sigma}(I(\mathbf{x})-l), \quad \text { where } \quad H=\sum_{l=I_{\min }}^{I_{\max }} h(l),
$$

with $h(I)$ denoting the histogram of observed (discrete) intensities $I \in\left[I_{\min }, I_{\max }\right]$.

Combining the new local intensity term for the outside and the region-based term for the inside yields the energy:

$$
E_{I}(\phi)=-\int_{\Omega} \alpha H(\phi) \log p_{\text {in }}(I(\mathbf{x}) \mid \phi)+(1-\alpha)(1-H(\phi)) \log p_{\text {out }}(I(\mathbf{x}) \mid \mathbf{x}) d \mathbf{x},
$$

while the role of the weights $\alpha$ and $(1-\alpha)$ are to balance those opposing forces which typically occur in slightly different value ranges.

With regards to the local curvature models, we determined the local mean curvatures as described in Sec. 3.2 for each organ. By this we obtained curvature maps which reflect the different convex and concave region of each organ, see Fig. 2(c).

\subsection{Global Shape Prior and Final Approach}

Although the spatially modulated curvature term imposes local constraints on the shape, in order to increase robustness of the overall segmentation approach, we also add prior information based on a global statistical shape model. Specifically, we follow a similar approach as recently reported in [5], where projections $\hat{\phi}$ of the current level set map $\phi$ into a linear subspace of learned shape variations are used to guide the shape evolution, by adding a term of the form $E_{S}(\phi):=\int_{\Omega}(\phi-\hat{\phi})^{2} d \mathbf{x}$ to the overall energy. In our experiments, we used a liver model which was built on a training set of 100 liver and 20 right kidney shapes, respectively.

Finally, the overall segmentation algorithm amounts to minimize the weighted sum of intensity-dependent $E_{I}$, the curvature-dependent $E_{C}$ and the shape-priorbased energy $E_{S}$, by iteratively descending along the negative energy gradient, which is represented by the partial differential equation:

$$
\frac{\partial E}{\partial \phi}=\delta_{\epsilon}(\phi)\left(\omega_{I}\left(\alpha \log p_{\text {in }}(I \mid \mathbf{x})-\beta \log p_{\text {out }}(I \mid \phi)\right)+\omega_{C}(\mathbf{x}) \operatorname{div} \frac{\nabla \phi}{|\nabla \phi|}+\omega_{S}(\phi-\hat{\phi})\right) \text {. }
$$

Thereby, the key points $\left\{\mathbf{x}_{i}\right\}$ are updated according to (1) in every iteration in parallel.

In our experiments, $\phi$ was initialized by the reference shape of the local features models, which were manually aligned with the organ in question with regards to translation and rotation. The latter can easily be automatized by an atlas registration technique, or a Pictorial structure based approach, for example. 

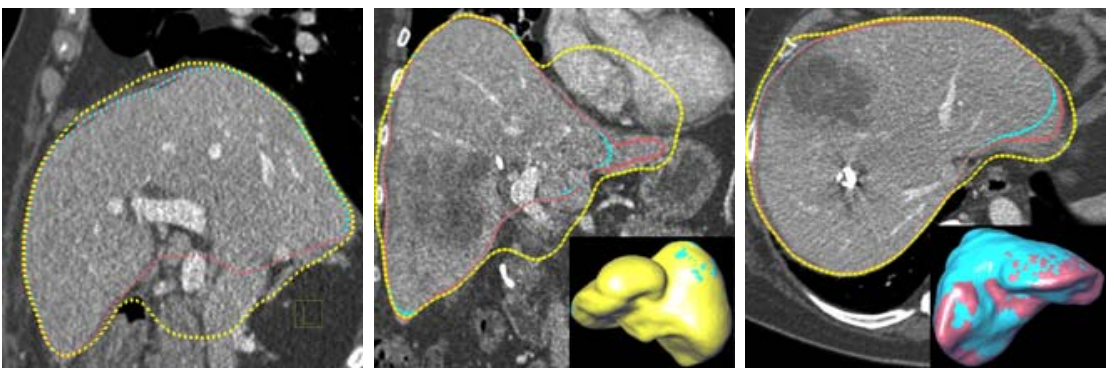

Fig. 3. Qualitative comparison of a standard approach with adaptive Parzen density estimation of the background intensities, cf. 4, and a global mean curvature force, cf. [2], (yellow) against the proposed local intensity prior (blue) and the locally modulated area penalization term in addition (red).

\section{Experiments}

Besides qualitative comparisons of the new local feature priors versus existing global approaches (see Fig. 3), we evaluated our algorithm on the 20 (mostly pathological) CT liver cases from the MICCAI '07 Liver challenge [7, and for the right kidney on 13 other CT cases which were annotated by own experts. None of them were included in the training sets. The reference shape of the local feature models served as initial shapes, which were manually aligned to each organ. The iteration were let run until convergence. All parameter values were the very same for all cases, except for a slightly weaker global curvature weight $\xi$ in the kidney cases. The obtained volumetric and surface-based errors in Table1 1 show that our proposed method produces state-of-the-art results, especially w.r.t. the average surface distance error of only $1.46 \mathrm{~mm} / 0.88 \mathrm{~mm}$ for the liver/kidney, respectively. In addition, the fact that these results were gained without adjusting none of the involved parameter to any individual data set clearly shows the high degree of generalization and, given the strong variability of liver shapes and appearances in the test set, the high robustness of the presented algorithm.

Table 1. Volumetric and surface errors, cf. [7, as well as scores for the 20 training data sets of the Liver 2007 Challenge, as well as for 13 other CT right kidney data sets

\begin{tabular}{|c|c|c|c|c|c|c|}
\hline Mean/std.dev. & $\begin{array}{l}\text { Overlap. } \\
\text { Err. [\%] }\end{array}$ & $\begin{array}{c}\text { Volume } \\
\text { Diff. [\%] }\end{array}$ & $\begin{array}{l}\text { Avg. Surf. } \\
\text { Dist. [mm] }\end{array}$ & $\begin{array}{l}\text { RMS Surf. } \\
\text { Dist. [mm] }\end{array}$ & $\begin{array}{l}\text { Maximum } \\
\text { Dist. }[\mathrm{mm}]\end{array}$ & $\begin{array}{c}\text { Challenge } \\
\text { Score }\end{array}$ \\
\hline & $8.44 \pm 1.7$ & $3.94 \pm 2.72$ & $1.46 \pm 0.38$ & 2.9 & 3.34 & $\overline{66.6}$ \\
\hline 13 r.kidney c & $10.6 \pm 1.9$ & $4.82 \pm 3.44$ & $0.88 \pm 0.23$ & $1.92 \pm 0.52$ & $15.31 \pm 4.51$ & $72.8 \pm 7.3$ \\
\hline
\end{tabular}

\section{Conclusion}

In conclusion, we have developed a new method for general organ segmentation with level sets that incorporates local intensity statistics and local curvature by means of an efficient point-based tracking mechanism. We have shown that using 
level set methods enables us to incorporate recent advances in shape regularized segmentation. We have achieved results that are both qualitatively and quantitatively strong on some of the most challenging problems in organ segmentation.

In practice, we have not found point correspondence problems to effect the performance of the algorithm, however for future work we wish to investigate the effect of the tangential component of the tracked points. Proper treatment of this task would require ensuring correct correspondence, which is not explicitly guaranteed even by the method of [14.

In addition, we wish to investigate limitations of uni-modal statistical models employed by the tracked points, and whether using multi-modal or more sophisticated methods would yield improved segmentation.

Acknowledgments. We would like to thank Haibin Ling for helpful discussions and Bogdan Georgescu for providing some of the annotations we used.

\section{References}

1. Arya, S., Mount, D.M., Netanyahu, N.S., Silverman, R., Wu, A.Y.: An optimal algorithm for approximate nearest neighbor searching fixed dimensions. J. ACM 45(6), 891-923 (1998)

2. Chan, T., Vese, L.: Active contours without edges. T-IP 10(2), 266-277 (2001)

3. Cootes, T.F., Taylor, C.J., Cooper, D.H., Graham, J.: Active shape models - their training and applications. CVIU 61(1), 38-59 (1995)

4. Cremers, D., Rousson, M., Deriche, R.: A review of statistical approaches to level set segmentation: integrating color, texture, motion and shape. IJCV 72(2), 195215 (2007)

5. Farzinfar, M., Xue, X., Teoh, E.K.: Joint parametric and non-parametric curve evolution for medical image segmentation. In: Forsyth, D., Torr, P., Zisserman, A. (eds.) ECCV 2008, Part I. LNCS, vol. 5302, pp. 167-178. Springer, Heidelberg (2008)

6. Heimann, T., Münzing, S., Meinzer, H., Wolf, I.: A shape-guided deformable model with evolutionary algorithm initialization for 3D soft tissue segmentation. In: Karssemeijer, N., Lelieveldt, B. (eds.) IPMI 2007. LNCS, vol. 4584, pp. 1-12. Springer, Heidelberg (2007)

7. Heimann, T., van Ginneken, B., Styner, M., et al.: Comparison and evaluation of methods for liver segmentation from ct datasets. In: T-MI (in press, 2009)

8. Kainmueller, D., Lange, T., Lamecker, H.: Shape constrained automatic segmentation of the liver based on a heuristic intensity model. In: Proc. MICCAI Workshop on 3D Segmentation in the Clinic: A Grand Challenge (2007)

9. Lee, J., Kim, N., Lee, H., Seo, J.B., Won, H.J., Shin, Y.M., Shin, Y.G.: Efficient liver segmentation exploiting level-set speed images with $2.5 \mathrm{D}$ shape propagation. In: MICCAI Workshop on 3D Segmentation in the Clinic (2007)

10. Leventon, M.E., Grimson, W.E., Faugeras, O.: Statistical shape influence in geodesic active contours. In: CVPR 2000, vol. 1, pp. 1316-1323. IEEE, Los Alamitos (2000)

11. Ling, H., Zhou, S.K., Zheng, Y., Georgescu, B., Sühling, M., Comaniciu, D.: Hierarchical, learning-based automatic liver segmentation. In: CVPR (2008) 
12. Okada, T., Shimada, R., Sato, Y., Hori, M., Yokota, K., Nakamoto, M., Chen, Y., Nakamura, H., Tamura, S.: Automated segmentation of the liver from 3d ct images using probabilistic atlas and multi-level statistical shape model. In: Ayache, N., Ourselin, S., Maeder, A. (eds.) MICCAI 2007, Part I. LNCS, vol. 4791, pp. 86-93. Springer, Heidelberg (2007)

13. Piovano, J., Papadopoulo, T.: Local statistic based region segmentation with automatic scale selection. In: Forsyth, D., Torr, P., Zisserman, A. (eds.) ECCV 2008, Part II. LNCS, vol. 5303, pp. 486-499. Springer, Heidelberg (2008)

14. Pons, J.-P., Hermosillo, G., Keriven, R., Faugeras, O.: How to deal with point correspondences and tangential velocities in the level set framework. In: ICCV, pp. 894-899. IEEE, Los Alamitos (2003)

15. Tsai, A., Yezzi, A., Wells, W., Tempany, C., Tucker, D., Fan, A., Grimson, W.E., Willsky, A.: A shape-based approach to the segmentation of medical imagery using level sets. IEEE Trans. Medical Imaging 22(2), 137-154 (2003)

16. Wimmer, A., Soza, G., Hornegger, J.: Two-stage semi-automatic organ segmentation framework using radial basis functions and level sets. In: Proc. MICCAI Workshop on 3D Segmentation in the Clinic: A Grand Challenge (2007) 